Recent Insights into the Physics of the Sun and Heliosphere:

Highlights from SOHO and Other Space Missions

IAU Symposium, Vol. 203, 2001

P. Brekke, B. Fleck, and J. B. Gurman eds.

\title{
The Observation and Study of Two Prominence Events in the Infrared
}

\author{
W. D. $\mathrm{Cao}^{1,2}$ B. X. Ye $\mathrm{Ye}^{1,3}$ and W.Livingston ${ }^{4}$
}

\begin{abstract}
1.National Astronomical Observatories, Chinese Academy of Sciences, Beijing 100012, China

2.Yunnan Astronomical Observatory, Chinese Academy of Sciences, Kunming 650011, China

3.Beijing Astronomical Observatory, Chinese Academy of Sciences, Beijing 100012, China

4. National Solar Observatory, P. O. Box 26732, Tucson, AZ 85726, U.S.A.
\end{abstract}

\begin{abstract}
This paper reports the first extremely near infrared observation of two prominence events on Jan. 8, 1999 and Feb. 9, 1999 at $\mathrm{H}$ Pfund $\beta(46525 \AA)$ and $\mathrm{H}$ Bracket $\alpha(40512 \AA)$ using the McMath Telescope. We scanned the spectrograph slit across the prominences parallel to the limb to obtain three-dimensional data cubes (two spatial dimensions and one spectral dimension). By fitting these observed lines, we can directly determine some important physical parameters such as Doppler width $\left(\Delta \lambda_{D}\right)$, the optical thickness at line center $\left(\tau_{\lambda 0}\right)$, and the line displacement $\left(\Delta \lambda_{0}\right)$. Combined with simultaneous observation of Balmer $H_{\alpha}, H_{\beta}$, and $\mathrm{Ca} \mathrm{K}$ lines, the new results of structure and dynamics of limb prominence is presented. These results imply that these infrared lines can penetrate through the prominence and become a potential probe and tool, while $H_{\alpha}$ and $H_{\beta}$ sense the prominence skin only.
\end{abstract}

\section{Introduction}

With the rapid development of the infrared detector and the observation technology in this wavelength band, the infrared solar observation and research are attracting more and more solar physicists and become the hot topic in the present ground-based solar observation. A number of old solar physics problems, such as sunspot, filament, plage, network, and intranetwork, etc, were studied and observed renewedly in the infrared, many new and interesting results were discovered (Solanki et al. 1992; Rueedi et al. 1992,1998; Bruls et al. 1995; Lin et al. 1998; Muglach et al. 1992;). This paper reports the first extremely near infrared observation of two prominence events on Jan. 8, 1999 and Feb. 9, 1999 at $\mathrm{H}$ Pfund $\beta(46525 \AA)$ and $\mathrm{H}$ Bracket $\alpha(40512 \AA)$ using the McMath Telescope.

\section{Observations}

The two prominence events were observed on the horizontal IR spectrograph of McMath Telescope on Kitt Peak. The solar image is about $81.5 \mathrm{~cm}$ in diameter and its revolution scale is 2.39 " $/ \mathrm{mm}$. Input slit is parallel to the limb and its size is $0.5 \mathrm{~mm} \times 2.0 \mathrm{~mm}$. With the solar image fixed and under guider control, we began a couple of $\mathrm{mm}$ on the disk and stepped radially out in steps of $1 \mathrm{~mm}$. 
Except for atmospheric dispersion effects, each line should cover the same parts of the prominence, neglecting evolution.

In order to avoid the rapid evolution, we selected two quiescent prominences as samples of our observations. The lowest trace was where we guessed the prominence started above the chromosphere. The slit was moved radially off the limb along the prominence. Each step is about 2.39".

On Jan. 8, 1999, a quiescent prominence appeared in the northwest of the disk. We employed the horizontal spectrograph to observe it at mutil-series of hydrogen line. The spectral profiles of each step at $\mathrm{H}$ Balmer $\beta(4861 \AA), \mathrm{H}$ Balmer $\alpha(6563 \AA), \mathrm{H}$ Bracket $\alpha(40512 \AA), \mathrm{H}$ Pfund $\beta(46525 \AA)$ are obtained(Cao et al. 2000). These figures give us the impression that the prominence is optically thick in $\mathrm{H}$ Balmer $\beta$ and $\mathrm{H}$ Balmer $\alpha$, and optically thin in the other two infrared wavelength bands.

We took an observation of the other quiescent prominence at $\mathrm{CaK}(3934 \AA)$, H Balmer $\alpha(6563 \AA)$ and H Bracket $\alpha(40512 \AA)$ on Feb. 9, 1999. Cao(Cao et al. 2000) gives the spectral profiles of each step at these wavelength bands. Obviously, the prominence is mostly optically thin.

\section{Discussion and Result}

Assuming that $C D, \operatorname{Max}$ and $\mathrm{Min}$ represent the continuum value of the center disk, the maximum and the minimum of the spectrum in AD units, we defined a parameter $B$ as follows,

$$
B=\frac{M a x-M i n}{C D}
$$

Thus, if we know the spectral energy distribution for sun's disk center, we can grasp the absolute intensity of the prominence line emission. Table 1 show the parameter B of the two prominence events at the different wavelength, the data on Feb. 9 are denoted with asterisk.

If we suppose that the source function $S_{\lambda}$ of the prominence is contant, the intensity of the emission line can be given by

$$
I_{\lambda}=S_{\lambda}\left[1-\exp \left(-\tau_{\lambda}\right)\right]
$$

where $\tau_{\lambda}$ is th optical depth. Generally, the intensity at every point of the observational line profile can be expressed as

$$
I_{\lambda}=f\left(X, \lambda_{j}\right)=f\left(x_{1}, x_{2}, \ldots, x_{n}, \lambda_{j}\right)
$$

where $x_{1}, x_{2}, \ldots x_{n}$ are independent variables which correspond to $n$ independent fitting parameters. Assuming that theoretical line profile is $f\left(X, \lambda_{j}\right)$, then

$$
\psi(X)=\sum_{j=1}^{m}\left[f\left(X, \lambda_{j}\right)-I_{\lambda}\right]^{2}
$$

must be a minimum according to the principle of the least-square method. So, letting $X=\left(S_{\lambda}, \tau_{\lambda 0}, \Delta \lambda_{0}, \Delta \lambda_{D}\right)$, we fit all line profile of the two prominence using the following formulae,

$$
\tau_{\lambda}=\tau_{\lambda 0} e^{-\nu^{2}}, \nu=\frac{\lambda-\lambda_{0}-\Delta \lambda_{0}}{\Delta \lambda_{D}}
$$


and obtain $S_{\lambda}, \tau_{\lambda 0}, \Delta \lambda_{0}, \Delta \lambda_{D}$ of every line at the different wavelength band and scanning location.

From Table.1 and fitting analysis to the two prominence events, we can obtain the following primary results,

1. For the optically thick prominence on Jan. 8, H Bracket $\alpha(40512 \AA)$ could be brighter than $\mathrm{H}$ Balmer $\alpha(6563 \AA)$. However, it is converse for the optically thin prominence on Feb. 9.

2. For the optically thick prominence on Jan. $8, \Delta \lambda_{D}$ at $H_{\alpha}, H_{\beta}$, and $H B_{\alpha}$ range from 0.5 to $0.67 \AA$, from 0.9 to $1.2 \AA$, and from 2.6 to $3.4 \AA$, respectively. If we don't take the non-thermal turbulent velocity into account, the kinetic temperture values derived from $\Delta \lambda_{D}$ at $H_{\alpha}$ and $H_{\beta}$ are much higher that at $H B_{\alpha}$ and $H P_{\beta}$. However, for the optically thin prominence on Feb. 9, the kinetic temperture derived from $\Delta \lambda_{D}$ is almost same at $H_{\alpha}, C a K$, and $H B_{\alpha}$.

$\mathrm{H}$ Bracket $\alpha$ and $\mathrm{H}$ Pfund $\beta$ penetrate through the prominenc, while $\mathrm{H}$ Balmer $\alpha$ and $\mathrm{H}$ Balmer $\beta$ sense the prominence skin only. So the infrared spectral lines can be a very powerful tool and probe to study prominence.

Table 1. The parameter $B$ of the two prominence events.

\begin{tabular}{llllllll}
\hline \hline Step & $H_{\alpha}$ & $H_{\beta}$ & $H B_{\alpha}$ & $H P_{\beta}$ & $\mathrm{CaK}^{*}$ & $\mathrm{H}^{*}{ }_{\alpha}$ & $\mathrm{HB}_{\alpha}^{*}$ \\
\hline 1 & 0.149 & 0.073 & 0.306 & 0.058 & 0.16 & 0.1260 & 0.0169 \\
2 & 0.177 & 0.092 & 0.306 & 0.058 & 0.18 & 0.0814 & 0.0095 \\
3 & 0.193 & 0.107 & 0.293 & 0.078 & 0.18 & 0.0614 & 0.0086 \\
4 & 0.198 & 0.104 & 0.276 & 0.073 & 0.18 & 0.0704 & 0.0127 \\
5 & 0.192 & 0.098 & 0.278 & 0.077 & 0.18 & 0.0841 & 0.0132 \\
6 & 0.188 & 0.091 & 0.233 & 0.073 & 0.17 & 0.0863 & 0.0139 \\
7 & 0.178 & 0.086 & 0.204 & 0.075 & 0.16 & 0.0850 & 0.0133 \\
8 & 0.167 & 0.082 & 0.193 & 0.066 & 0.16 & 0.0834 & 0.0148 \\
9 & 0.157 & 0.076 & 0.134 & 0.055 & 0.16 & 0.0813 & 0.0180 \\
10 & 0.147 & 0.074 & 0.218 & 0.061 & 0.16 & 0.0748 & 0.0198 \\
11 & 0.138 & 0.070 & 0.163 & 0.060 & 0.14 & 0.0672 & 0.0165 \\
12 & 0.128 & 0.065 & 0.230 & 0.094 & 0.13 & 0.0477 & 0.0152 \\
13 & 0.124 & 0.059 & 0.244 & 0.089 & 0.14 & 0.0403 & 0.0105 \\
14 & 0.107 & 0.054 & 0.262 & 0.095 & 0.14 & 0.0426 & 0.0096 \\
15 & 0.086 & 0.046 & 0.256 & 0.094 & 0.13 & 0.0390 & 0.0097 \\
16 & 0.075 & 0.038 & 0.194 & 0.085 & 0.12 & 0.0424 & 0.0068 \\
17 & 0.061 & 0.028 & 0.106 & 0.058 & 0.12 & 0.0401 & 0.0059 \\
18 & 0.034 & 0.016 & 0.027 & 0.032 & 0.12 & 0.0290 & 0.0061 \\
19 & 0.034 & 0.011 & 0.054 & 0.011 & 0.12 & 0.0254 & 0.0075 \\
\hline
\end{tabular}

\section{References}

Bruls, J. H., Solanki, S. K., 1995, A\&A, 293, 240

Cao, W.D., Livingston, W., 2000, Publ. of Yunnan Obser., No.4, 35

Lin, H, Penn, M. J., Kuhu, J. R., 1998, Ap. J., 493, 978

Muglach, K., Solanki, S. K., 1992, A\&A, 263, 301

Rueedi, I., Solanki, S. K., Livingston, W., 1992, A\&A, 263, 323

Rueedi, I., Solanki, S. K., Keller, C. U., Frutiger, C., 1998, A\&A, 338, 1089

Solanki, S. K., Rueedi, I. K., Livingston, W., 1992, A\&A, 263, 312 